\title{
Development of a High-Fidelity, 3D Printed Otoplasty Surgical Simulator
}

\author{
Chelsey Wallace M.S. ${ }^{1}$, Zahra Nourmohammadi Ph.D. ${ }^{2}$, David A. Zopf M.D., \\ M.S. ${ }^{3,4}$ \\ ${ }^{1}$ Indiana University School of Medicine, ${ }^{2}$ University of Michigan Medical School, \\ ${ }^{3}$ University of Michigan, Department of Otolaryngology, ${ }^{4}$ University of Michigan, \\ Department of Biomedical Engineering
}

Background and Hypothesis: Protruding ears, also known as prominauris, are prevalent worldwide at an occurrence rate of about $5 \%$. Children with prominauris report lower self-esteem and experience increased teasing and social isolation at school. From a functional standpoint, protruding ears can make wearing prescription glasses difficult. This increased stress and anxiety and impaired functionality lead children and families to seek treatment. One of the most effective treatments for protruding ears is otoplasty. This procedure involves an incision in the back of the ear and the placement of non-resorbable sutures to reform the ear. Alternatively, the procedure can be performed using an incisionless technique. As this is an elective procedure done commonly in children, adequate education of medical trainees is critical to ensure the proper level of skill is attained and patient satisfaction is maximized.

Currently, teaching otoplasty is done with cadavers and supervised procedures with an attending. Surgical simulators are employed in the instruction of a variety of surgical procedures and allow residents to practice in a zero-risk environment. In addition, 3D printing has facilitated the development of surgical simulators allowing for a more cost-effective, consistent, and anatomically correct simulator. We developed an ear model made from silicone for trainees to practice traditional and incisionless otoplasty.

Project Methods: The otoplasty surgical simulator was developed by isolating an ear from a computed-tomography scan in the Materialise software to create a 3D model. This model was then altered to create a negative mold. The mold was printed using fusion deposition printing with $1.75 \mathrm{MM}$ polylactic acid filament. After printing, the mold was filled with Dragon Skin Silicone Shore 20 to simulate ear cartilage. The model was then coated in a layer of Dragon Skin Silicone Shore 10 to simulate a layer of skin.

Conclusion and Potential Impact: This otoplasty simulator will next be validated by expert surgeons and then used in a surgical simulation workshop for surgical trainees. Because of the low-cost of the surgical simulator and the ease of manufacturing, this simulator can also be used to train surgeons abroad where access to surgical training may not be readily available. 